\title{
Importance of Vesicle Release Stochasticity in Neuro-spike Communication
}

\author{
Hamideh Ramezani, Student Member, IEEE and Ozgur B. Akan, Fellow, IEEE
}

\begin{abstract}
Aim of this paper is proposing a stochastic model for vesicle release process, a part of neuro-spike communication. Hence, we study biological events occurring in this process and use microphysiological simulations to observe functionality of these events. Since the most important source of variability in vesicle release probability is opening of voltage dependent calcium channels (VDCCs) followed by influx of calcium ions through these channels, we propose a stochastic model for this event, while using a deterministic model for other variability sources. To capture the stochasticity of calcium influx to presynaptic neuron in our model, we study its statistics and find that it can be modeled by a distribution defined based on Normal and Logistic distributions.
\end{abstract}

\section{INTRODUCTION}

Understanding communication theoretical capabilities of information transmission among neurons, known as neurospike communication, is a significant step in developing bioinspired nano-networking solutions [1]. For this aim, the impact of biological processes included in this communication on its performance should be recognized. In the literature, two extremes can be found for modeling these processes, (i) strongly simplified deterministic ordinary differential equations, (ii) most detailed biophysiological models called microphysiological models [2], which attempt to model reality as accurate as possible. Somewhere in-between, we find more simple stochastic models including impacts of the most important elements while ignoring others. Focus of this study is deriving such a model for vesicle release process.

The influx of calcium ions upon arrival of a spike to the pre-synaptic terminal is necessary for releasing vesicles that contain neurotransmitters, i.e., information carriers in neuro-spike communication [3]. Trial to trial variations in calcium concentration upon spike arrival affects the vesicle release probability. These fluctuations arise from (i) opening of VDCCs, (ii) binding of calcium ions to buffers, calcium sensors and pumps, (iii) diffusion of calcium ions and buffers [4]. In this paper, we simulate the microphysiological model of vesicle release process in 3D using Monte Carlo methods (MCell version 3 [5], [6] ${ }^{1}$ ). Moreover, we use a deterministic model to achieve the average concentration of calcium ions

H. Ramezani is with the Next-Generation and Wireless Communications Laboratory, Department of Electrical and Electronics Engineering, Koç University, Istanbul 34450, Turkey (e-mail: hramezani13@ku.edu.tr).

O. B. Akan is with the Internet of Everything (IoE) Group, Electrical Engineering Division, Department of Engineering, University of Cambridge, Cambridge CB2 1PZ, U.K. (e-mail: oba21@cam.ac.uk).

This work was supported in part by ERC project MINERVA (ERC-2013CoG \#616922), EU project CIRCLE (EU-H2020-FET-Open \#665564), and TŬBİTAK graduate scholarship program (BIDEB-2215)

${ }^{1}$ MCell development is supported by the NIGMS-funded (P41GM103712) National Center for Multiscale Modeling of Biological Systems (MMBioS).

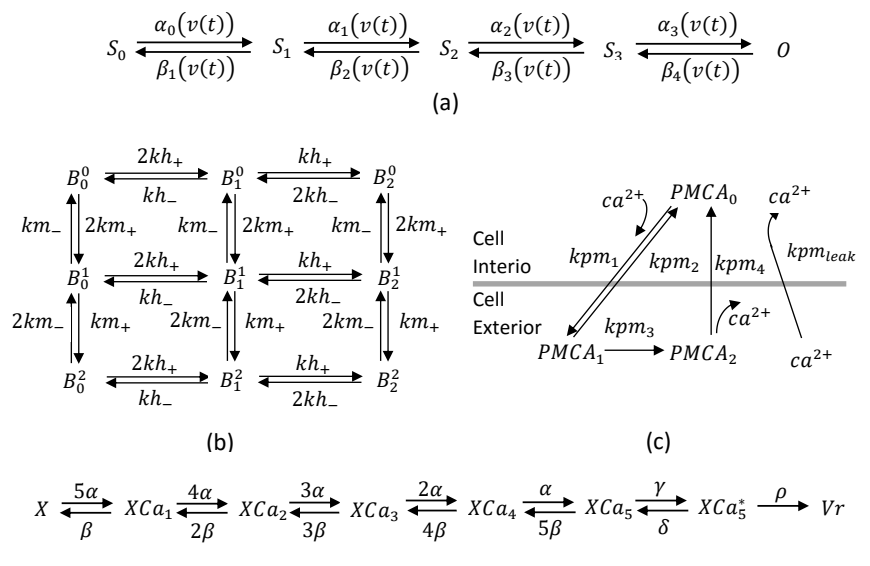

(d)

Fig. 1. Kinetic scheme of (a) VDCCs gating, (b) Calbindin buffer, (c) PMCA pump, and (d) calcium sensor.

inside pre-synaptic terminal. Then, since the fluctuations in influx of calcium ions through opened VDCCs has a strong effect on the vesicle release probability [4], we consider these fluctuations as noise and achieve their statistics by microphysiological simulations. We observe that using $\mathrm{Bi}$ nomial distribution for modeling opening of VDCCs, which is suggested in the literature [7], is insufficient for catching the stochasticity of calcium influx process. Moreover, we find that Normal and Logistic distributions can be used to model the stochasticity of calcium influx process.

The remainder of this paper is organized as follows. In Section II, the biological background of the vesicle release process is presented. Using kinetic schemes, a deterministic model for average changes in calcium concentration is derived in Section III. Then, statistics of calcium influx process is studied in Section IV, where fluctuations of calcium influx rate is modeled by a random process whose distribution is defined based on VDCCs gating and Normal and Logistic distributions. Finally, the accuracy of the model is evaluated in Section V and the paper is concluded in Section VI.

\section{BIOLOGICAL BACKGROUND}

Arrival of a spike to the axonal terminal changes its membrane potential, which, in turn, affects the opening of VDCCs. The kinetic scheme of VDCCs gating is given in Fig. 1(a) [4], where $v(t)$ is the membrane potential at pre-synaptic terminal, $\left\{S_{0}, S_{1}, S_{2}, S_{3}\right\}$ are closed states and $O$ is the open state. Voltage-dependent forward and backward rates between states are calculated by $\alpha_{i}(v(t))=\alpha_{i} \exp \left(\frac{v(t)}{V_{i+1}}\right)$ and $\beta_{i}(v(t))=\beta_{i} \exp \left(\frac{-v(t)}{V_{i}}\right)$, 
where $\alpha_{i}, \beta_{i}$, and $V_{i}$ for $i \in\{1,2,3,4\}$ are constants related to the type of VDCCs. When the membrane potential changes are strong enough, the VDCCs open and allow the entering of calcium ions to the pre-synaptic terminal. Amount of spike evoked calcium influx through an open VDCC can be calculated by

$$
C a_{i n}^{2+}(t)=0.5 e^{-1} g(55 \mathrm{mV}-v(t)),
$$

where $g$ and $e$ are the conductance of VDCCs and the elementary charge, respectively [8]. After entering the presynaptic terminal, the calcium ions diffuse inside the terminal, where they may bind to (i) buffers such as Calbindin, which can bind up to 4 calcium ions as shown in Fig. 1(b), (ii) the PMCA pumps, whose kinetic scheme is shown in Fig. 1(c), and (iii) the calcium sensor that can bind up to 5 calcium ions as shown in Fig. 1(d) [9]. Note that when a buffer is in state $B_{j}^{i}$ for $0 \leq i, j \leq 2, i+j$ calcium ions are bound to it and when calcium sensor is in state $X C a_{i}$ it has $i$ calcium ions bound to it. Moreover, when the sensor enters the state $V r$, a vesicle releases.

\section{Deterministic Modeling of Vesicle Release}

We model a segment of pre-synaptic terminal in Hippocampal pyramidal neurons by a rectangular box with $0.5 \mu m$ width and $4 \mu m$ length [9]. Moreover, we discretize the time and pre-synaptic domain by steps defined as $\Delta t$ and $\Delta \vec{x}$, where $\Delta \vec{x}=\left[\Delta x_{1}, \Delta x_{2}, \Delta x_{3}\right]^{\prime}$. Then, we calculate the concentration of Calbindin buffers, PMCA pumps, calcium sensors, and calcium ions in each voxel of this domain as $\vec{B}(\vec{x}, t), \vec{P}(\vec{x}, t), \overrightarrow{C s}(\vec{x}, t)$, and $C a(\vec{x}, t)$, respectively.

a) Calbindin Buffers: The concentration of Calbindin buffers changes by, (i) their binding to calcium ions and (ii) diffusion of them inside pre-synaptic terminal. Impacts of reaction between Calbindin and calcium ions on concentration of Calbindin buffers is derived by

$$
\frac{\partial \vec{B}(\vec{x}, t)}{\partial t}=\boldsymbol{B}_{\boldsymbol{F}} C a(\vec{x}, t) \vec{B}(\vec{x}, t)+\boldsymbol{B}_{\boldsymbol{B}} \vec{B}(\vec{x}, t),
$$

where $\vec{B}(\vec{x}, t)$ is given by (3), $B_{j}^{i}(\vec{x}, t)$ is the concentration of Calbindin buffer in state $B_{j}^{i}$ at place $\vec{x}$ and time $t$, and matrices $\boldsymbol{B}_{\boldsymbol{F}}$ and $\boldsymbol{B}_{\boldsymbol{B}}$, given by (4), (5), contain forward and backward rate of reactions shown in Fig. 1(b), respectively.

Diffusion of Calbindin buffers inside the pre-synaptic domain is defined based on the diffusion equation as

$$
\frac{\partial \vec{B}(\vec{x}, t)}{\partial t}=D_{c b} \sum_{i=1}^{3} \frac{\partial^{2} \vec{B}(\vec{x}, t)}{\partial x_{i}^{2}} ; \quad \vec{x} \in \Omega, t>0,
$$

where $D_{c b}$ is diffusion coefficient of Calbindin buffers and $\Omega$ corresponds to inside of pre-synaptic terminal, i.e., $-x_{i, \max } \leq x_{i} \leq x_{i, \max }$ for $i \in\{1,2,3\}$ with $x_{1, \max }=$ $\frac{2 \mu \mathrm{m}}{\Delta x_{1}}, x_{2, \max }=\frac{0.25 \mu \mathrm{m}}{\Delta x_{2}}$, and $x_{3, \max }=\frac{0.25 \mu \mathrm{m}}{\Delta x_{3}}$. Calbindin buffers cannot pass boundaries of pre-synaptic terminal, hence we use the Neumann boundary conditions as follows.

$\frac{\partial \vec{B}(\vec{x}, t)}{\partial x_{i}}=0 ; \quad x_{i}= \pm x_{i, \max }, \forall x_{j}, j \neq i, j \in\{1,2,3\}$.

Using finite difference method, (6) is approximated by

$$
\begin{aligned}
& \frac{\vec{B}(\vec{x}, t+\Delta t)-\vec{B}(\vec{x}, t)}{\Delta t}= \\
& D_{c b} \sum_{i=1}^{3} \frac{\vec{B}\left(\vec{x}+e_{i}, t\right)-2 \vec{B}(\vec{x}, t)+\vec{B}\left(\vec{x}-e_{i}, t\right)}{\Delta x_{i}^{2}},
\end{aligned}
$$

where $e_{i}$ is the $3 \times 1$ vector with one at $i$ th entry and zero at other entries. Using backward difference at boundary conditions with $x_{i}=-x_{i, \max }$ and forward difference at boundary conditions with $x_{i}=x_{i, \max }$, we reach

$$
\begin{aligned}
\vec{B}\left(\vec{x}-e_{i}, t\right)=\vec{B}(\vec{x}, t) ; & x_{i}=-x_{i, \max } \\
\vec{B}\left(\vec{x}+e_{i}, t\right)=\vec{B}(\vec{x}, t) ; & x_{i}=x_{i, \max } .
\end{aligned}
$$

$$
\begin{aligned}
& \vec{B}(\vec{x}, t)=\left[B_{0}^{0}(\vec{x}, t), B_{1}^{0}(\vec{x}, t), B_{2}^{0}(\vec{x}, t), B_{0}^{1}(\vec{x}, t), B_{1}^{1}(\vec{x}, t), B_{2}^{1}(\vec{x}, t), B_{0}^{2}(\vec{x}, t), B_{1}^{2}(\vec{x}, t), B_{2}^{2}(\vec{x}, t)\right]^{\prime} \\
& \boldsymbol{B}_{\boldsymbol{F}}=-\left[\begin{array}{ccccccccc}
2 k m_{+}+2 k h_{+} & 0 & 0 & 0 & 0 & 0 & 0 & 0 & 0 \\
-2 k m_{+} & k m_{+}+2 k h_{+} & 0 & 0 & 0 & 0 & 0 & 0 & 0 \\
0 & -k m_{+} & 2 k h_{+} & 0 & 0 & 0 & 0 & 0 & 0 \\
-2 k h_{+} & 0 & 0 & 2 k m_{+}+k h_{+} & 0 & 0 & 0 & 0 & 0 \\
0 & -2 k h_{+} & 0 & -2 k m_{+} & k m_{+}+k h_{+} & 0 & 0 & 0 & 0 \\
0 & 0 & -2 k h_{+} & 0 & -k m_{+} & k h+ & 0 & 0 & 0 \\
0 & 0 & 0 & -k h_{+} & 0 & 0 & 2 k m_{+} & 0 & 0 \\
0 & 0 & 0 & 0 & -k h_{+} & 0 & -2 k m_{+} & k m_{+} & 0 \\
0 & 0 & 0 & 0 & 0 & -k h_{+} & 0 & -k m_{+} & 0
\end{array}\right] \\
& \boldsymbol{B}_{\boldsymbol{B}}=-\left[\begin{array}{ccccccccc}
0 & -k m_{-} & 0 & -k h_{-} & 0 & 0 & 0 & 0 & 0 \\
0 & k m_{-} & -2 k m_{-} & 0 & -k h_{-} & 0 & 0 & 0 & 0 \\
0 & 0 & 2 k m_{-} & 0 & 0 & -k h_{-} & 0 & 0 & 0 \\
0 & 0 & 0 & k h_{-} & -k m_{-} & 0 & -2 k h_{-} & 0 & 0 \\
0 & 0 & 0 & 0 & k m_{-}+k h_{-} & -2 k m_{-} & 0 & -2 k h_{-} & 0 \\
0 & 0 & 0 & 0 & 0 & k h_{-}+2 k m_{-} & 0 & 0 & -2 k h_{-} \\
0 & 0 & 0 & 0 & 0 & 0 & 2 k h_{-} & -k m_{-} & 0 \\
0 & 0 & 0 & 0 & 0 & 0 & 0 & 2 k h_{-}+k m_{-} & -2 k m_{-} \\
0 & 0 & 0 & 0 & 0 & 0 & 0 & 0 & 2 k h_{-}+2 k m_{-}
\end{array}\right]
\end{aligned}
$$


Based on (2), (6), and (7), the concentration of Calbindin buffers at $t+\Delta t$ is achieved by

$$
\begin{aligned}
& \vec{B}(\vec{x}, t+\Delta t)=\vec{B}(\vec{x}, t)+ \\
& \Delta t \times D_{c b} \sum_{i=1}^{3} \frac{\vec{B}\left(\vec{x}+e_{i}, t\right)-2 \vec{B}(\vec{x}, t)+\vec{B}\left(\vec{x}-e_{i}, t\right)}{\Delta x_{i}^{2}}+ \\
& \Delta t \times\left(\boldsymbol{B}_{\boldsymbol{F}} C a(\vec{x}, t) \vec{B}(\vec{x}, t)+\boldsymbol{B}_{\boldsymbol{B}} \vec{B}(\vec{x}, t)\right) .
\end{aligned}
$$

b) PMCA Pumps and Calcium Sensors: The impact of binding of calcium ions to PMCA pumps and calcium sensors on their concentration is achieved same as (2). Then, using finite difference method, the concentration of PMCA pumps and calcium sensors at time $t+\Delta t$ is derived as

$$
\begin{gathered}
\vec{P}(\vec{x}, t+\Delta t)=\vec{P}(\vec{x}, t)+ \\
\Delta t \times\left(\boldsymbol{P}_{\boldsymbol{F}} C a(\vec{x}, t) \vec{P}(\vec{x}, t)+\boldsymbol{P}_{\boldsymbol{B}} \vec{P}(\vec{x}, t)\right), \\
\overrightarrow{C s}(\vec{x}, t+\Delta t)=\overrightarrow{C s}(\vec{x}, t)+ \\
\Delta t \times\left(\boldsymbol{C s}_{\boldsymbol{F}} C a(\vec{x}, t) \overrightarrow{C s}(\vec{x}, t)+\boldsymbol{C s}_{\boldsymbol{B}} \overrightarrow{C s}(\vec{x}, t)\right),
\end{gathered}
$$

where $\boldsymbol{P}_{\boldsymbol{F}}$ and $\boldsymbol{C} \boldsymbol{s}_{\boldsymbol{F}}$ contain the forward rate of reactions of calcium ions with PMCA pumps and sensors, respectively, and $\boldsymbol{P}_{\boldsymbol{B}}$ and $\boldsymbol{C} \boldsymbol{s}_{\boldsymbol{B}}$ contain backward rate of these reactions. These four matrices are achieved based on the kinetic schemes given by Fig. 1(c) and Fig. 1(d) with same manner used to find $\boldsymbol{B}_{\boldsymbol{F}}$ and $\boldsymbol{B}_{\boldsymbol{B}}$.

c) Calcium Ions: Calcium ions enter the pre-synaptic terminal from open VDCCs. A deterministic model for VDCCs opening is derived by considering their kinetic scheme as Markov model. Then, state equations are achieved by

$$
\begin{array}{rrr}
\frac{d S_{0}(t)}{d t}= & & -\alpha_{0}(v(t)) S_{0}(t)+\beta_{1}(v(t)) S_{1}(t) \\
\frac{d S_{i}(t)}{d t}= & & \alpha_{i-1}(v(t)) S_{i-1}(t)-\beta_{i}(v(t)) S_{i}(t)- \\
& \alpha_{i}(v(t)) S_{n}(t)+\beta_{i+1}(v(t)) S_{i+1}(t) \\
\frac{O(t)}{d t}= & \alpha_{3}(v(t)) S_{3}(t)-\beta_{4}(v(t)) O(t) .
\end{array}
$$

where $i \in\{1,2,3\}$. By solving (9)-(11) for spike shape, we can derive average probability of having an open VDCC of a given type, $O(t)$. Then, the calcium influx at each time step from VDCC located at $\vec{x}_{V D C C}$ is found by using (1) as

$$
H_{V D C C}\left(\vec{x}_{V D C C}, t+\Delta t\right)=n_{c h} O(t) C a_{i n}^{2+}(t) \Delta t .
$$

Binding to buffers, pumps, sensors and diffusion of calcium ions are other factors that affect the concentration of these ions. Hence, the concentration of calcium ions at time
$t+\Delta t$ is derived by (12), where $\boldsymbol{B}_{\boldsymbol{F}, \boldsymbol{i \boldsymbol { i }}}, \boldsymbol{B}_{\boldsymbol{B}, \boldsymbol{i \boldsymbol { i }}}, \boldsymbol{P}_{\boldsymbol{F}, \boldsymbol{i \boldsymbol { i }}}, \boldsymbol{P}_{\boldsymbol{B}, \boldsymbol{i \boldsymbol { i }}}$, $\boldsymbol{C} \boldsymbol{s}_{\boldsymbol{F}, \boldsymbol{i i}}$, and $\boldsymbol{C} \boldsymbol{s}_{\boldsymbol{B}, \boldsymbol{i} \boldsymbol{i}}$ are the diagonal entries of these matrices and $\vec{B}_{i}(\vec{x}, t), \vec{P}_{i}(\vec{x}, t)$, and $\vec{C} s_{i}(\vec{x}, t)$ are the $i$ th entry of these vectors. Note that same as (8), we use the Neumann boundary conditions for calcium concentration at boundaries since these ions cannot pass boundaries.

d) Vesicle Release: When the Calcium sensor is in state $S_{5}^{*}$ with rate $\rho$ releases the vesicle that is attached to it. Hence, the average concentration of released vesicles at time $t+\Delta t$ is derived by $\operatorname{Vr}(\vec{x}, t+\Delta t)=\rho S_{5}^{*}(\vec{x}, t) \Delta t+\operatorname{Vr}(\vec{x}, t)$.

\section{Stochasticity in Release Process}

In this section, we propose a stochastic model for vesicle release based on the deterministic model introduced in Section III and results of the microphysiological model.

\section{A. Microphysiological model of Vesicle Release}

We place VDCCs in a cluster at the center of one face of the box used to model a segment of pre-synaptic terminal. To achieve the rates of the uni-molecular reactions among states of VDCCs, we use a piece-wise linear approximation of spike shape as given in Fig. 2. Initially, PMCA pumps are uniformly distributed on boundaries, Calbindin buffers and $100 \mathrm{nM}$ calcium ions are uniformly distributed inside the pre-synapse. Using parameters tabulated in Table I, we simulate following events in MCell, (i) VDCCs opening, (ii) diffusion of calcium ions and calcium buffers inside presynaptic terminal, (iii) binding of calcium ions to calcium buffers, (iv) pumping of calcium ions to cell exterior by calcium pumps, and (v) triggering of vesicle release by binding of calcium ions to the calcium sensor.

\section{B. Stochastic Model of Vesicle Release}

The variation of calcium influx through open VDCCs is the most important source of variability in vesicle release process. Moreover, impact of other variability sources on vesicle release probability are negligible [4]. Hence, we propose a stochastic model for calcium influx rate upon arrival of a spike and use the deterministic models described in Section III for other processes.

The opening of each VDCC is suggested to be modeled by Bernoulli distribution with mean $O(t)$ [7]. However, by comparing the standard deviation (STD) of the calcium influx rate achieved by using this distribution and microphysiological simulation shown in Fig. 3, we conclude that Binomial distribution does not model the stochasticity of calcium influx process accurately.

$$
\begin{aligned}
& C a(\vec{x}, t+\Delta t)= C a(\vec{x}, t)+H_{V D C C}(\vec{x}, t+\Delta t) \\
& \Delta t \times\left[\sum_{i=1}^{9}\left(\boldsymbol{B}_{\boldsymbol{F}, \boldsymbol{i i}} C a(\vec{x}, t) \vec{B}_{i}(\vec{x}, t)+\boldsymbol{B}_{\boldsymbol{B}, \boldsymbol{i i}} \vec{B}_{i}(\vec{x}, t)\right)+\sum_{i=1}^{3}\left(\boldsymbol{P}_{\boldsymbol{F}, \boldsymbol{i}} C a(\vec{x}, t) \vec{P}_{i}(\vec{x}, t)+\boldsymbol{P}_{\boldsymbol{B}, \boldsymbol{i i}}, \vec{P}_{i}(\vec{x}, t)\right)+\right. \\
&\left.\quad \sum_{i=1}^{6}\left(\boldsymbol{C s}_{\boldsymbol{F}, \boldsymbol{i} \boldsymbol{i}} C a(\vec{x}, t) \vec{C} s_{i}(\vec{x}, t)+\boldsymbol{C s}_{\boldsymbol{B}, \boldsymbol{i i}} \vec{C} s_{i}(\vec{x}, t)\right)+D_{C a} \sum_{i=1}^{3} \frac{C a\left(\vec{x}+e_{i}, t\right)-2 C a(\vec{x}, t)+C a\left(\vec{x}-e_{i}, t\right)}{\Delta x_{i}^{2}}\right]
\end{aligned}
$$


TABLE I

PARAMETERS FOR VESICLE RELEASE MODEL

\begin{tabular}{|c|c|c|c|}
\hline Parameter & Symbol & Value & Ref. \\
\hline \multirow{2}{*}{ Spike shape parameters } & $w, t_{\text {peak }}$ and $t_{\text {under }}$, & $4,0.5 \mathrm{~ms}$ and $w / 2$ & \multirow{2}{*}{ [9], [10] } \\
\hline & $V_{\text {rest }}, V_{\text {peak }}$ and $V_{\text {under }}$ & $-65,40$ and $-80 \mathrm{mV}$ & \\
\hline The elementary charge & $e$ & $1.602 \times 10^{-19} \mathrm{C}$ & \\
\hline \multirow{3}{*}{ VDCCs gating parameters } & $\left\{\alpha_{0}, \alpha_{1}, \alpha_{2}, \alpha_{3}\right\}$ & $\{4.04,6.7,4.39,17.33\} \mathrm{ms}^{-1}$ & \multirow{3}{*}{ [4] } \\
\hline & $\left\{\beta_{1}, \beta_{2}, \beta_{3}, \beta_{4}\right\}$ & $\{2.88,6.39,8.16,1.84\} \mathrm{ms}^{-1}$ & \\
\hline & $\left\{V_{1}, V_{2}, V_{3}, V_{4}\right\}$ & $\{49.14,42.08,55.31,26.55\} \mathrm{mV}$ & \\
\hline Conductance and Number of VDCCs & $g$ and $n_{c h}$ & $2.7 \mathrm{pS}$ and 22 & [8], [4] \\
\hline Calcium and Calbindin diffusion constant & $D_{C a}$ and $D_{c b}$ & $220 \mu \mathrm{m}^{2} / \mathrm{s}$ and $28 \mu \mathrm{m}^{2} / \mathrm{s}$ & [9] \\
\hline Resting intracellular calcium and Calbindin concentration & & $100 \mathrm{nM}$ and $45 \mu \mathrm{M}$ & [9] \\
\hline \multirow{2}{*}{ Calbindin kinetic parameters } & $k h_{+}, k h_{-}$ & $0.55 \times 10^{7} \mathrm{M}^{-1} \mathrm{~s}^{-1}, 2.6 \mathrm{~s}^{-1}$ & \multirow{2}{*}{ [9] } \\
\hline & $k m_{+}, k m_{-}$ & $4.35 \times 10^{7} \mathrm{M}^{-1} \mathrm{~s}^{-1}, 35.8 \mathrm{~s}^{-1}$ & \\
\hline PMCA surface density & & $180 \mu \mathrm{m}^{-2}$ & [9] \\
\hline \multirow{2}{*}{ PMCA kinetic parameters } & $k p m_{1}, k_{p m}$ & $1.5 \times 10^{7} \mathrm{M}^{-1} \mathrm{~s}^{-1}, 20 \mathrm{~s}^{-1}$ & \multirow{2}{*}{ [9] } \\
\hline & $k p m_{3}, k_{p m}, \mathrm{kpm}_{\text {leak }}$ & $20 \mathrm{~s}^{-1}, 100 \mathrm{~s}^{-1}, 12.5 \mathrm{~s}^{-1}$ & \\
\hline \multirow{2}{*}{ Calcium sensor kinetic parameters } & $\alpha, \beta$ & $0.3 \mu \mathrm{M}^{-1} \mathrm{~ms}^{-1}, 3 \mathrm{~ms}^{-1}$ & \multirow{2}{*}{ [4] } \\
\hline & $\gamma, \delta, \rho$ & $30 \mathrm{~ms}^{-1}, 8 \mathrm{~ms}^{-1}, 40 \mathrm{~ms}^{-1}$ & \\
\hline
\end{tabular}

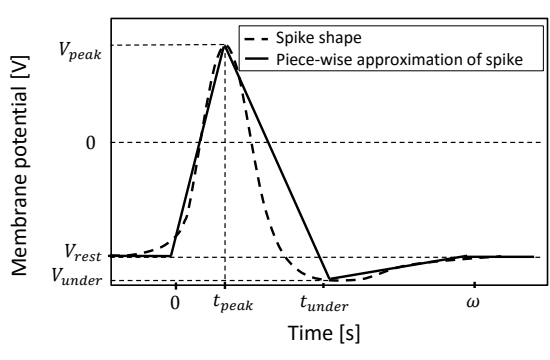

Fig. 2. Approximation of spike shape.

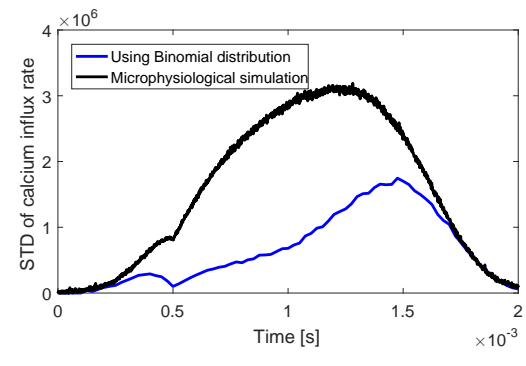

Fig. 3. STD of calcium influx rate.

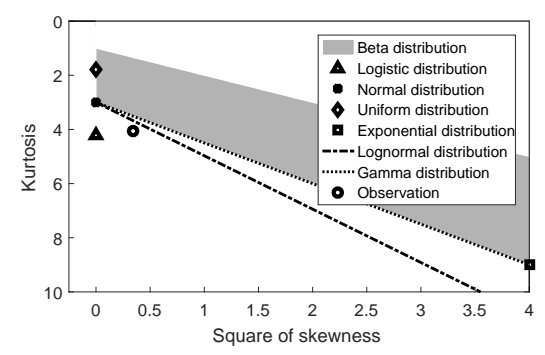

Fig. 4. Cullen and Frey graph.
Since we can achieve the average rate of calcium influx from VDCCs based on the deterministic model explained in Section III, we consider variations of this rate as channel noise and derive its statistics using results of microphysiological simulation. To find candidate distributions for modeling this noise, we use Cullen and Frey graph, in which the ratio of kurtosis to square of skewness of data is compared to this ratio for various standard distributions [11]. Based on Fig. 4 and the fact that our noise can get negative values, we examine the goodness of fitting the channel noise by Normal and Logistic distributions. For this aim, we use the quantilequantile (Q-Q) and probability-probability (P-P) plots as shown in Fig. 5 and conclude that Normal distribution can be used to model the probability density function (pdf) for negative channel noises while the Logistic distribution fits to pdf for positive noises. Hence, we define $f\left(x(t) \mid \sigma_{n}(t), s_{p}(t)\right)$ as pdf for channel noise with parameters $\sigma_{n}(t)$ and $s_{p}(t)$ as $f\left(x(t) \mid \sigma_{n}(t), s_{p}(t)\right)=\left\{\begin{array}{ll}f_{N}\left(x(t) \mid \mu(t), \sigma_{n}(t)\right) ; & x \leq \mu \\ f_{L}\left(x(t) \mid \mu(t), s_{p}(t)\right) ; & x>\mu\end{array}\right.$,

where $\sigma_{n}(t)$ is the STD of negative noises and $s_{p}(t)$ is the scale parameter of the Logistic distribution, which can be found by $\frac{\sqrt{3} \sigma_{p}(t)}{\pi}$ based on STD of positive noises, i.e., $\sigma_{p}(t) . f_{N}\left(x(t) \mid \mu(t), \sigma_{n}(t)\right)$ and $f_{L}\left(x(t) \mid \mu(t), s_{p}(t)\right)$ are pdf of Normal and Logistic distributions whose mean and STD are given by $\mu(t), \sigma_{n}(t)$ and $\mu(t), \sigma_{p}(t)$, respectively. We model STD of negative and positive noises for each VDCC type as $\sigma_{n}(t)=a_{n} \sqrt{O(t) C a_{i n}^{2+}(t)}$ and $\sigma_{p}(t)=$ $a_{p} \sqrt{O(t) C a_{i n}^{2+}(t)}$, where $n_{c h} O(t) C a_{i n}^{2+}(t)$ is the average rate of the calcium influx. Hence, both average of calcium influx rate and parameters of channel noise are defined based on the kinetic scheme of VDCCs. $a_{n}=250$ and $a_{p}=350$ are derived based on data achieved by microphysiological simulation with parameters given in Table I. Then, we derive $\mu(t)$ such that the mean of our proposed distribution becomes 0 , hence,

$$
\mu(t)=\int_{-\infty}^{0} y f_{N}\left(y \mid 0, \sigma_{n}(t)\right) d y+\int_{0}^{\infty} y f_{L}\left(y \mid 0, s_{p}(t)\right) d y .
$$

\section{Experimental evaluation}

We utilize the Kullback Leibler distance, i.e., $D_{K L}(P, Q)$, which measures the distance between two probability distributions given by $P(x)$ and $Q(x)$ to evaluate the accuracy of calcium influx statistics achieved by our model.

$$
D_{K L}(P, Q)=\int P(x) \log _{2}\left(\frac{P(x)}{Q(x)}\right)+Q(x) \log _{2}\left(\frac{Q(x)}{P(x)}\right) d x
$$




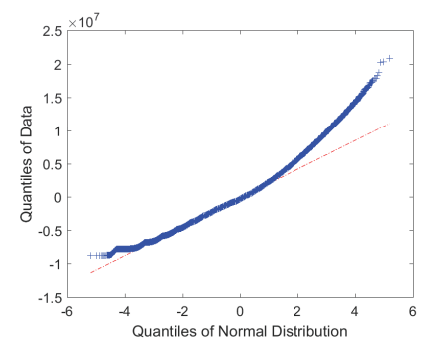

(a)

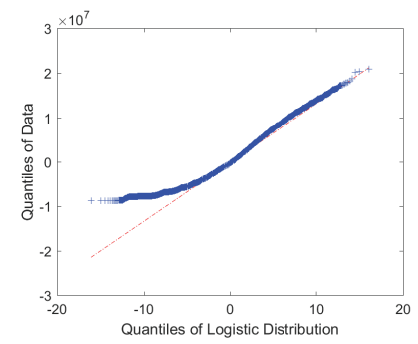

(b)

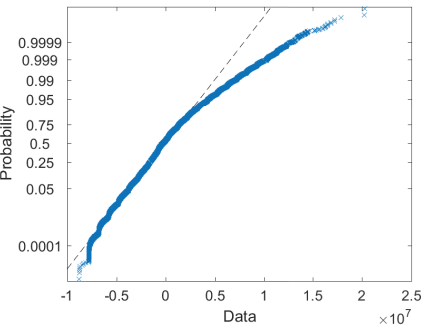

(c)

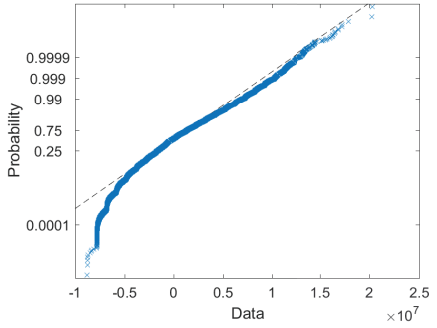

(d)

Fig. 5. (a) and (b) Q-Q plots, (c) and (d) P-P plots for fitting channel noise with Normal and Logistic distributions, respectively.

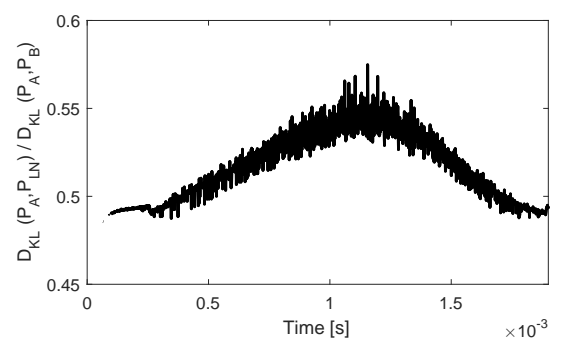

(a)

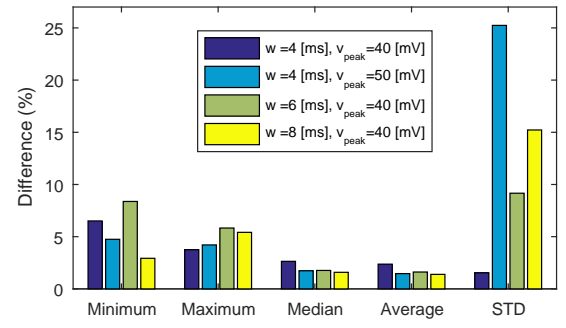

(b) Proposed stochastic model.

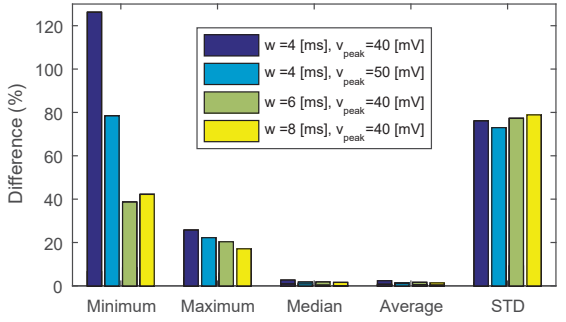

(c) Using Binomial distribution for VDCCs gating.

Fig. 6. Experimental results, (a) ratio of Kullback Leibler distances for the proposed distribution and the existing one in the literature, (b) and (c) normalized difference between the statistics of total calcium ions entered the pre-synaptic terminal achieved by microphysiological simulations and stochastic models.

Using this metric, we evaluate changes of $\frac{D_{K L}\left(P_{A}, P_{L N}\right)}{D_{K L}\left(P_{A}, P_{B}\right)}$ after spike arrival to pre-synaptic terminal, where $P_{A}$ is the actual distribution of channel noise, $P_{L N}$ and $P_{B}$ are the distributions derived by our proposed model and using Binomial distribution for VDCCs opening, respectively. This ratio is depicted in Fig. 6(a) for parameters given in Table I, which shows that our proposed stochastic model outperforms the existing model in the literature. Moreover, we use different spike shapes, i.e, spikes with various $v_{\text {peak }}$ and $w$ [12], and examine the accuracy of our model for some of statistics of total calcium ions entered the pre-synaptic terminal. As shown in Fig. 6(b), changes in the spike shape does not significantly affect the accuracy of first order statistics of the total calcium ions entered to the neuron achieved by our stochastic model, while the difference between the STD of the total calcium ions achieved by our model and microphysiological simulation is sensitive to the spike shape variations. However, based on Fig. 6, the maximum difference between these statistics is less than $\% 26$ for our proposed model, which shows a significant improvement compared to the results achieved by existing model in the literature.

\section{CONCLUSION}

In this paper, we propose a stochastic model for vesicle release process, in which trial to trial variability in calcium influx is considered as channel noise. By studying statistics of this noise based on the data derived by microphysiological simulations, we investigate that the negative noises can be fitted by a Normal distribution, while fitting a Logistic distribution to the possitive ones is more appropriate. Hence, we define a distribution based on Normal and Logistic distributions and derive the parameters of this distribution based on the kinetic of VDCCs. By comparing the statistics of channel noises generated by our stochastic model and microphysiological simulations, we conclude that proposed distribution captures stochasticity of channel noise more accurately compared to the existing model in the literature.

\section{REFERENCES}

[1] O. B. Akan et al., "Fundamentals of molecular information and communication science," Proceedings of the IEEE, vol. 105, no. 2 , pp. 306-318, 2017.

[2] J. R. Stiles et al., "Synaptic variability: new insights from reconstructions and monte carlo simulations," Synapses, vol. 1, pp. 681-731, 2001.

[3] K. R. Delaney and E. E. Stanley, "Calcium and neurotransmitter release," eLS, 2001.

[4] C. Modchang et al., "A comparison of deterministic and stochastic simulations of neuronal vesicle release models," Physical biology, vol. 7 , no. 2, p. $026008,2010$.

[5] J. R. Stiles et al., "Monte carlo methods for simulating realistic synaptic microphysiology using mcell," Computational neurosci.: realistic modeling for experimentalists, pp. 87-127, 2001.

[6] R. A. Kerr et al., "Fast monte carlo simulation methods for biological reaction-diffusion systems in solution and on surfaces," SIAM J. on scientific computing, vol. 30, pp. 3126-3149, 2008.

[7] J. A. White et al., "Channel noise in neurons," Trends in neurosci. vol. 23, no. 3, pp. 131-137, 2000.

[8] Y. S. Ermolyuk et al., "Differential triggering of spontaneous glutamate release by p/q-, n-and r-type ca2+ channels," Nature neurosci., vol. 16, no. 12, pp. 1754-1763, 2013.

[9] S. Nadkarni et al., "Modelling vesicular release at hippocampal synapses," PLoS Comput Biol, vol. 6, no. 11, p. e1000983, 2010.

[10] M. F. Bear et al., Neuroscience: Exploring the Brain, 3rd ed., 2007.

[11] A. C. Cullen and H. C. Frey, Probabilistic techniques in exposure assessment: a handbook for dealing with variability and uncertainty in models and inputs. Springer Science \& Business Media, 1999.

[12] H. Ramezani and O. B. Akan, "A communication theoretical modeling of axonal propagation in hippocampal pyramidal neurons," IEEE Trans. NanoBiosci., 2017. 